\title{
Inadequate feeding of infant and young children in India: lack of nutritional information or food affordability?
}

\author{
Nisha Malhotra* \\ Faculty of Arts, University of British Columbia, 997-1873 East Mall, Vancouver, British Columbia, \\ Canada, V6T 1 Z1
}

Submitted 18 February 2012: Final revision received 28 June 2012: Accepted 26 July 2012: First published online 3 September 2012

\begin{abstract}
Objective: Despite a rapidly growing economy and rising income levels in India, improvements in child malnutrition have lagged. Data from the most recent National Family Health Survey reveal that the infant and young child feeding (IYCF) practices recommended by the WHO and the Indian Government, including the timely introduction of solid food, are not being followed by a majority of mothers in India. It is puzzling that even among rich households children are not being fed adequately. The present study analyses the socioeconomic factors that contribute to this phenomenon, including the role of nutritional information.

Design: IYCF practices from the latest National Family Health Survey (2005-2006) were analysed. Multivariate logistic regression analyses were performed to establish the determinants of poor feeding practices. The indicators recommended by the WHO were used to assess the IYCF practices.

Setting: India.

Subjects: Children ( $n$ 9241) aged 6-18 months.

Results: Wealth was shown to have only a small effect on feeding practices. For children aged 6-8 months, the mother's wealth status was not found to be a significant determinant of sound feeding practices. Strikingly, nutritional advice on infant feeding practices provided by health professionals (including anganwadi workers) was strongly correlated with improved practices across all age groups. Exposure to the media was also found to be a significant determinant. Conclusions: Providing appropriate information may be a crucial determinant of sound feeding practices. Efforts to eradicate malnutrition should include the broader goals of improving knowledge related to childhood nutrition and IYCF practices.
\end{abstract}

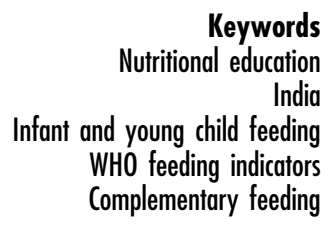

In India, an alarming $43 \%$ of children less than 3 years of age are stunted, $48 \%$ are underweight and $17 \%$ are wasted, according to the latest National Family Health Survey (NFHS; 2005-2006) ${ }^{(1)}$. It is well known that poor anthropometric indices of children have serious consequences for their survival. Nutritional intake in the early years of life can also have long-term health consequences in adulthood. Lower nutritional intake is positively associated with an increased risk of $\mathrm{CHD}$, lower intellectual and poor reproductive performance ${ }^{(2-6)}$. Malnutrition in childhood can also have intergenerational impacts. A mother's poor nutritional intake and growth during her infancy is associated with lower birth weight and lower risk of survival for her offspring ${ }^{(3,7-10)}$

A balanced diet rich in both macro- and micronutrients is fundamental to an infant's growth and development ${ }^{(11)}$. According to current WHO recommendations on infant and young child feeding (IYCF), an appropriate diet is breast-feeding for the first 6 months of life, followed by adequate and nutritionally balanced solid food to complement breast milk $^{(12)}$. The introduction of solid or semi-solid food at around 6 months of age is crucial, since breast milk alone would no longer be sufficient for maintaining a child's optimal growth ${ }^{(13-16)}$. Generally, there is a clustering effect of nutritional deficiency, so that a few nutrients cannot be singled out, and a wellbalanced diet is vital for children. Diversity in an infant's diet has been linked to improved nutritional status and anthropometric measures in childhood ${ }^{(17,18)}$.

Food requirements vary across breast-feeding status. According to the guidelines ${ }^{(19)}$, a child in the age range 6-23 months should be fed from four or more different food groups in addition to breast milk. Further, a child who does not receive any breast milk should be fed from four or more food groups and should also receive milk or milk products on a daily basis. In its indicators for assessing IYCF 
practices in population-based surveys, the WHO classifies seven food groups: (i) Milk and Milk Products; (ii) Eggs; (iii) Flesh Foods (Meat, Fish, etc.); (iv) Vitamin-A Rich Fruits and Vegetables; (v) Other Fruits and Vegetables; (vi) Grains; (vii) Nuts and Legumes. The Indian National Guidelines on IYCF (based on WHO's guiding principles) also recommend that after the first 6 months of age children should no longer be exclusively breast-fed, and should be given complementary solid and semi-solid food ${ }^{(20,21)}$.

Breast-feeding is nearly universal in India where $96 \%$ of children younger than 5 years of age are breast-fed; the mean duration of breast-feeding is 24.7 months $^{(9)}$. Nevertheless, there are prolonged delays in the introduction of complementary food, with only $55 \cdot 8 \%$ of children aged $6-9$ months receiving such food. Further, it is during this time of delay that the incidence of childhood malnutrition rises in the population ${ }^{(9,22,23)}$. This is also true of other developing countries; malnutrition rates rise during 6-8 months of age, coinciding with complementary feeding ${ }^{(24)}$.

Much of the research on this issue has emphasized poverty as a leading cause of malnutrition ${ }^{(25-28)}$. Income is a binding constraint to proper nutrition; however, income is only one of the many determinants of poor feeding practices. Maternal characteristics like formal schooling, wealth and employment are also found to be important in mothers' timely introduction of complementary food ${ }^{(29-31)}$.

The present study uses data from the NFHS (2005-2006), which provides useful information on IYCF practices at the national level, to evaluate the extent to which various factors previously found to be important determinants of poor feeding practices apply to the case of India. The study also highlights the role of nutrition information, in comparison to formal education and wealth, as an important determinant of infant diet. This work contributes by separating and quantifying the contribution of nutritional information and informal education to the prevalence of inadequate feeding practices.

\section{Methods}

\section{Data}

NFHS (2005-2006) data are used for the present analysis ${ }^{(32)}$; NFHS is the Indian component of the Measure Demographic and Health Survey project. NHFS provides a cross-sectional data set. The survey was nationally representative and collected information from 124385 eligible women aged 15-49 years in all of India's twentyeight states. The survey collected information on their children born in the three years preceding the survey. Survey data include demographic and health parameters as well as information on food consumed by these children.

\section{Outcome measure: complementary feeding}

All children aged 6-18 months were included in the analysis. Data on children's dietary intake were obtained from mothers, using the conventional $24 \mathrm{~h}$ recall method. Mothers were also asked about the daily frequency of feeds (number of feeds in $24 \mathrm{~h}$ ) and the various food groups offered in the meal. Inadequate feeding here is defined as falling short of the WHO guidelines, as evident by the late introduction of complementary food, low meal frequency, or insufficient food groups or quantity of complementary food. The IYCF indicators as suggested in the WHO guidelines for population-based surveys ${ }^{(19)}$ are used. These indicators reflect three aspects of IYCF practices: timely introduction of solid/semi-solid foods, minimum dietary diversity and minimum meal frequency. For children in the age group 6 to 8 months, the WHO indicators are: complementary food (child was offered any solid/semi-solid food); child was fed two or more times daily; and child was offered food from four or more food groups. Given that only 3\% of the sample was offered food from four or more food groups, another variable was introduced for this age range: child was offered food from at least three food groups. For young children in the age group 9-18 months the WHO indicators are: child was fed three or more times daily; child was offered food from three or more food groups; and child was offered food from four or more food groups.

Food groups were based on a $24 \mathrm{~h}$ recall of different food products fed to the child. Food group categories are comparable to those used in previous literature ${ }^{(12,13)}$ and are: milk, cheese, yoghurt or other milk products; bread, noodles or other food made from grains; oil, fats, butter or products made from them; mangoes, papaya or other vitamin-A-rich fruits; beans, legumes/lentils and nuts; other fruits and vegetables (including green leafy vegetables); and eggs, meat, poultry, fish and shellfish (and organ meats).

\section{Exposure measures: nutritional information and otber socio-economic variables}

Information variables were created using questions related to the mother's contact with health professionals and exposure to media. Information regarding nutrition and IYCF practices was considered separately from formal education. The NFHS survey asked questions on whether nutritional advice was provided during meetings with health professionals (in the previous three months) or during antenatal care visits. More specifically, the survey asked: 'In the last three months, have you met with an anganwadi worker or other community health worker?' and 'Did you see anyone for antenatal care for this (recent) pregnancy?'. Mothers were then asked about the different matters addressed in the meeting. Responses were divided into eighteen categories, one of which was 'Nutrition/Health Education and Nutrition Advice'. The anganwadi health professionals are the workers at anganwadi centres set up by the Indian Government under the Integrated Child Development Services to take care of the health needs of children and mothers ${ }^{(20)}$. 
Frequency of media exposure, i.e. how often a mother, in total, read newspapers, listened to the radio and watched television, was also included in the analysis. Values ranged from not at all (0), less than once a week (1), at least once a week (2) to almost every day (3). Mothers' formal education was categorized by the highest level attained: no education (the reference group), less than primary, primary, middle school and completed high school.

A number of other socio-economic variables were included in this analysis. Two binary variables pertaining to the mother's work situation were included, as these can affect the time she has available to feed a child and also reflects that she might obtain information in the workplace. First, if a mother currently worked (in a paid labour force, either at home or away from home), the variable took the value 1 , and 0 otherwise. A mother who is formally employed could either be working away from home (leaves the house to work outside) or could be working from home. The second variable pertains to the location of the mother's workplace; if a working mother (as defined above) had been working away from home, the variable took the value of 1 , and 0 if she worked from home. The NFHS data do not contain information on individual or household incomes, but do provide a wealth index, which is used in the present analysis. The wealth index was based on asset ownership and housing characteristics of each household (e.g. type of flooring; material of exterior walls; type of roofing; cooking fuel; house ownership; ownership of a bicycle, a motorcycle or scooter, an animal-drawn cart, a car, etc.). Principal component analysis was used to assign weights to each asset (housing characteristics), which was then standardized. For each household, asset scores were summed and individuals were ranked according to the household score. The wealth index was divided into quintiles for the whole sample: poorest (the reference group), poorer, middle, richer and richest.

The following variables were used to account for any gender bias or lack of experience in feeding practices: number of siblings the child has aged less than 3 years, gender (equals 1 if the child is male), first child (equals 1 if the child is first born) and rural (equals 1 if the household resides in a rural area).

\section{Statistical methods}

In total, six regression models were used to test for the relationship between various indicators of IYCF practices, as recommended by the WHO, and socio-economic variables (including nutritional information). The same covariates were used for all models and the differences across the models are based on the outcome measures: different indicators of IYCF practices. All these models were tested using logistic multivariate regression analysis. The odds ratio, adjusted for all the covariates, and 95\% confidence interval are reported for all regressions.
Three regression models were used for infants in the age group 6-8 months (Models 1 to 3 ) and these covered three binary outcome measures: complementary feeding, fed two or more times daily, and fed from three or more food groups. Three regression models (Models 4, 5 and 6) were used for children in the age group 9-18 months, and these covered three indicators measured as binary variables: complementary feeding, fed three or more times daily, and fed from four or more food groups. The STATA statistical software package version SE $11 \cdot 1$ was used for all statistical analyses and regressions.

\section{Results}

\section{Baseline statistics}

Table 1 lists the baseline characteristics of the survey participants included in the study, stratified by age group (6-8 months (Gp1) and 9-18 months (Gp2)). In Gp1 (Gp2), $99 \%(98 \%)$ of mothers had an antenatal check-up and $35 \%(32 \%)$ had met with a health-care worker in the three months preceding the survey. For both groups, 56\% of mothers discussed nutrition during antenatal check-ups, whereas only $1.5 \%$ of mothers in Gp1 and $1.8 \%$ of mothers in Gp2 discussed nutrition during other meetings with health-care workers. Among exposures to the media, television exposure was the highest, followed by radio and print media; this was true for both groups. Both groups were also comparable in terms of wealth index.

Table 2 reports descriptive statistics on feeding practices. In the sample, only $63 \%$ of children between the ages of 6 and 8 months were given any solid/semi-solid food; this rose to $84 \%$ of children between the ages of 9 and 11 months. Although $92 \%$ of children between the ages of 12 and 18 months in the sample received complementary food, only $17 \%$ of them were fed adequately from four or more food groups, and only $54 \%$ of them were fed three or more times daily.

\section{Multivariate regression}

The results of multivariate logistic regressions in Tables 3 and 4 provide evidence of a strong association between nutritional information and adequate IYCF practices.

Table 3 presents adjusted odds ratios and 95\% confidence intervals from the multivariate logistic regression analysis for children in the age group 6-8 months. For children aged 6-8 months, mothers were more likely to give complementary food to their child, offer food at least twice daily and from three or more food groups, if they had received nutritional advice during antenatal check-ups than if they had not $(\mathrm{OR}=1.48,1.29$ and 1.55 , respectively). This was true even for mothers who discussed nutrition during other meetings (non-antenatal) with health professionals: they were more likely to give complementary food $(\mathrm{OR}=3 \cdot 76)$. Exposure to radio increased meal frequency and diversity, and the likelihood of mothers offering 
Table 1 Descriptive statistics of the study population

\begin{tabular}{|c|c|c|c|c|c|c|}
\hline \multirow[b]{3}{*}{ Variable } & \multicolumn{6}{|c|}{ Age group } \\
\hline & \multicolumn{3}{|c|}{$6-8$ months } & \multicolumn{3}{|c|}{ 9-18 months } \\
\hline & $n$ & $\%$ & SD & $n$ & $\%$ & SD \\
\hline \multicolumn{7}{|l|}{ Mother's exposure to health-care professionals } \\
\hline Had antenatal check up & 2202 & $99 \cdot 46$ & 0.07 & 6869 & $97 \cdot 61$ & $0 \cdot 15$ \\
\hline Advised on nutrition (antenatal) & 1241 & $56 \cdot 05$ & 0.50 & 3971 & $56 \cdot 43$ & 0.50 \\
\hline Met health-care worker in the last 3 months & 772 & $34 \cdot 87$ & 0.48 & 2211 & $31 \cdot 42$ & 0.46 \\
\hline Discussed nutrition in last 3 months & 32 & $1 \cdot 45$ & $0 \cdot 12$ & 125 & $1 \cdot 78$ & $0 \cdot 13$ \\
\hline \multicolumn{7}{|l|}{ Exposure to media (ever) } \\
\hline Watched television & 1463 & $66 \cdot 08$ & 0.48 & 4627 & $65 \cdot 75$ & 0.47 \\
\hline Listened to radio & 988 & $44 \cdot 63$ & $0 \cdot 50$ & 2994 & $42 \cdot 55$ & 0.49 \\
\hline Read newspaper or magazine & 816 & $36 \cdot 86$ & 0.48 & 2467 & 35.06 & 0.48 \\
\hline \multicolumn{7}{|l|}{ Wealth index* } \\
\hline Poorest & 379 & $17 \cdot 12$ & $0 \cdot 38$ & 1193 & $16 \cdot 95$ & 0.38 \\
\hline Poorer & 399 & 18.02 & 0.38 & 1299 & $18 \cdot 46$ & 0.39 \\
\hline Middle & 453 & $20 \cdot 46$ & $0 \cdot 40$ & 1428 & $20 \cdot 29$ & 0.40 \\
\hline Richer & 513 & $23 \cdot 17$ & 0.42 & 1623 & 23.06 & 0.42 \\
\hline Richest & 470 & $21 \cdot 23$ & $0 \cdot 41$ & 1494 & $21 \cdot 23$ & 0.41 \\
\hline \multicolumn{7}{|l|}{ Mother's education } \\
\hline No education & 826 & $37 \cdot 31$ & 0.48 & 2640 & $37 \cdot 52$ & 0.48 \\
\hline Less than primary & 148 & $6 \cdot 68$ & $0 \cdot 25$ & 500 & $7 \cdot 11$ & 0.26 \\
\hline Primary school & 152 & $6 \cdot 87$ & 0.25 & 498 & $7 \cdot 08$ & 0.26 \\
\hline Middle school & 749 & $33 \cdot 83$ & 0.47 & 2436 & $34 \cdot 62$ & 0.48 \\
\hline High school and above & 339 & $15 \cdot 31$ & $0 \cdot 36$ & 963 & $13 \cdot 68$ & 0.34 \\
\hline \multicolumn{7}{|l|}{ Mother's employmentt } \\
\hline Currently working & 626 & $28 \cdot 27$ & 0.45 & 2285 & $32 \cdot 47$ & 0.47 \\
\hline Work: from home & 124 & $5 \cdot 60$ & 0.23 & 481 & $6 \cdot 84$ & 0.25 \\
\hline Work: away from home & 500 & $22 \cdot 58$ & 0.42 & 1798 & $25 \cdot 55$ & 0.44 \\
\hline \multicolumn{7}{|l|}{ Other variables } \\
\hline Male child & 1143 & $51 \cdot 63$ & 0.50 & 3645 & $51 \cdot 80$ & 0.50 \\
\hline Had diarrhoea recently & 411 & $18 \cdot 56$ & 0.39 & 1133 & $16 \cdot 10$ & 0.37 \\
\hline No. of siblings aged $<3$ years & 587 & $26 \cdot 51$ & 0.46 & 1205 & $17 \cdot 12$ & 0.39 \\
\hline First child & 744 & 33.60 & 0.47 & 2343 & $33 \cdot 30$ & 0.47 \\
\hline Low birth weight - subjective & 138 & $6 \cdot 23$ & 0.24 & 444 & $6 \cdot 31$ & 0.24 \\
\hline Total & 2214 & & & 7037 & & \\
\hline
\end{tabular}

Frequency, percentage and standard deviation of explanatory variables considered for the multivariate regression models, stratified by age group, from the 2005-2006 Indian National Family Health Survey.

*Wealth index was based on asset ownership and housing characteristics of the household; the five categories were based on wealth quintiles of the whole sample of households (whereas, in the present analysis, we only consider households with children $\leq 18$ months of age).

tFormal paid employment.

Table 2 Details on feeding practices

\begin{tabular}{|c|c|c|c|c|c|c|c|c|c|}
\hline \multirow[b]{3}{*}{ Variable } & \multicolumn{9}{|c|}{ Age group } \\
\hline & \multicolumn{3}{|c|}{$6-8$ months } & \multicolumn{3}{|c|}{ 9-11 months } & \multicolumn{3}{|c|}{$12-18$ months } \\
\hline & $n$ & $\%$ & SD & $n$ & $\%$ & SD & $n$ & $\%$ & SD \\
\hline $\begin{array}{l}\text { Complementary feeding } \\
\text { Meal frequency }\end{array}$ & 1395 & 63 & $0 \cdot 48$ & 1682 & 84 & $0 \cdot 37$ & 4632 & 92 & $0 \cdot 2$ \\
\hline $\begin{array}{l}\text { Two or more feeds daily } \\
\text { Three or more feeds daily }\end{array}$ & $\begin{array}{r}1129 \\
554\end{array}$ & $\begin{array}{l}51 \\
25\end{array}$ & $\begin{array}{l}0.50 \\
0.43\end{array}$ & $\begin{array}{r}1381 \\
781\end{array}$ & $\begin{array}{l}69 \\
39\end{array}$ & $\begin{array}{l}0.46 \\
0.49\end{array}$ & $\begin{array}{l}4330 \\
2719\end{array}$ & $\begin{array}{l}86 \\
54\end{array}$ & $\begin{array}{l}0.35 \\
0.50\end{array}$ \\
\hline Dietary diversity & & & & & & & & & \\
\hline $\begin{array}{l}\text { Three or more food groups } \\
\text { Four or more food groups }\end{array}$ & $\begin{array}{r}155 \\
66\end{array}$ & $\begin{array}{l}7 \\
3\end{array}$ & $\begin{array}{l}0 \cdot 26 \\
0 \cdot 18\end{array}$ & $\begin{array}{l}400 \\
180\end{array}$ & $\begin{array}{r}20 \\
9\end{array}$ & $\begin{array}{l}0 \cdot 40 \\
0 \cdot 28\end{array}$ & $\begin{array}{r}1762 \\
856\end{array}$ & $\begin{array}{l}35 \\
17\end{array}$ & $\begin{array}{l}0.48 \\
0.37\end{array}$ \\
\hline & $n$ & Mean & SD & $n$ & Mean & SD & $n$ & Mean & SD \\
\hline $\begin{array}{l}\text { No. of feeds/d } \\
\text { No. of food groups/d }\end{array}$ & $\begin{array}{l}2214 \\
2214\end{array}$ & $\begin{array}{l}1 \cdot 54 \\
0 \cdot 78\end{array}$ & $\begin{array}{l}1.53 \\
1.05\end{array}$ & $\begin{array}{l}2002 \\
2002\end{array}$ & $\begin{array}{l}2 \cdot 18 \\
1 \cdot 49\end{array}$ & $\begin{array}{l}\overline{1.54} \\
1.33\end{array}$ & $\begin{array}{l}\overline{5035} \\
5035\end{array}$ & $\begin{array}{l}2 \cdot 72 \\
2 \cdot 13\end{array}$ & $\begin{array}{l}1.45 \\
1.47\end{array}$ \\
\hline
\end{tabular}

Frequency, percentage, mean and standard deviation of feeding practices, as per the WHO feeding guidelines, stratified by age group of the child, from the 2005-06 Indian National Family Health Survey.

complementary food in that age group, food at least twice daily and from at least three out of the seven food groups $(\mathrm{OR}=1.08,1.12$ and 1.19 respectively; food groups: milk and milk products; food made from grains; oil, fats, butter or products made from them; vitamin-A-rich fruits; beans, legumes/lentils and nuts; other fruits and vegetables 
Table 3 Information and adequate feeding practices (6-8 months)

\begin{tabular}{|c|c|c|c|c|c|c|}
\hline & \multirow{2}{*}{\multicolumn{2}{|c|}{$\frac{\text { Complementary feeding (yes) }}{\text { Model } 1}$}} & \multirow{2}{*}{\multicolumn{2}{|c|}{$\frac{\text { Two or more times daily }}{\text { Model } 2}$}} & \multirow{2}{*}{\multicolumn{2}{|c|}{$\frac{\text { Three or more food groups }}{\text { Model } 3}$}} \\
\hline & & & & & & \\
\hline & OR & $95 \% \mathrm{Cl}$ & OR & $95 \% \mathrm{Cl}$ & OR & $95 \% \mathrm{Cl}$ \\
\hline \multicolumn{7}{|c|}{ Mother's exposure to health-care professionals } \\
\hline \multicolumn{7}{|c|}{ Had antenatal check up } \\
\hline No & $1 \cdot 00$ & Ref. & $1 \cdot 00$ & Ref. & $1 \cdot 00$ & Ref. \\
\hline Yes & $2 \cdot 00$ & $0.54,7 \cdot 42$ & $0.05^{\star}$ & $0.01,0.44$ & 0.43 & $0.05,3 \cdot 71$ \\
\hline \multicolumn{7}{|l|}{ Advised on nutrition (antenatal) } \\
\hline No & 1.00 & Ref. & $1 \cdot 00$ & Ref. & 1.00 & Ref. \\
\hline Yes & $1 \cdot 48^{*}$ & $1 \cdot 21,1 \cdot 82$ & $1 \cdot 29+$ & $1 \cdot 06,1 \cdot 58$ & $1 \cdot 55 t$ & $1 \cdot 03,2 \cdot 33$ \\
\hline \multicolumn{7}{|c|}{ Met with health-care worker in last 3 months } \\
\hline No & $1 \cdot 00$ & Ref. & $1 \cdot 00$ & Ref. & 1.00 & Ref. \\
\hline Yes & 0.95 & $0 \cdot 78,1 \cdot 15$ & $0 \cdot 88$ & $0.73,1.06$ & 0.94 & $0 \cdot 66,1 \cdot 34$ \\
\hline \multicolumn{7}{|l|}{ Discussed nutrition and health } \\
\hline No & $1 \cdot 00$ & Ref. & $1 \cdot 00$ & Ref. & $1 \cdot 00$ & Ref. \\
\hline Yes & $3 \cdot 76+$ & $1 \cdot 27,11 \cdot 13$ & $1 \cdot 88$ & $0 \cdot 87,4.08$ & $0 \cdot 70$ & $0 \cdot 16,3 \cdot 08$ \\
\hline \multicolumn{7}{|l|}{ Exposure to media } \\
\hline Watched television & 1.07 & $0 \cdot 97,1 \cdot 17$ & 1.04 & $0.95,1 \cdot 14$ & 1.08 & $0.91,1.29$ \\
\hline Listened to radio & $1 \cdot 08 \ddagger$ & $0.99,1 \cdot 17$ & $1 \cdot 12^{*}$ & $1 \cdot 03,1 \cdot 21$ & $1 \cdot 19+$ & $1 \cdot 04,1 \cdot 37$ \\
\hline Read newspaper or magazine & $1 \cdot 30^{\star}$ & $1 \cdot 13,1 \cdot 50$ & $1 \cdot 21^{\star}$ & $1 \cdot 06,1 \cdot 37$ & $1 \cdot 18 \ddagger$ & $0.97,1.44$ \\
\hline \multicolumn{7}{|l|}{ Wealth index } \\
\hline Poorest & 1.00 & Ref. & $1 \cdot 00$ & Ref. & 1.00 & Ref. \\
\hline Poorer & $0 \cdot 75 \ddagger$ & $0.56,1.00$ & $0 \cdot 77 \ddagger$ & $0.57,1.03$ & $0 \cdot 83$ & $0.41,1.65$ \\
\hline Middle & 0.88 & $0.65,1 \cdot 20$ & 0.90 & $0.66,1.23$ & 0.84 & $0.42,1.68$ \\
\hline Richer & $1 \cdot 14$ & $0.81,1.62$ & $1 \cdot 16$ & $0.83,1.64$ & $1 \cdot 45$ & $0.72,2 \cdot 94$ \\
\hline Richest & $1 \cdot 28$ & $0.83,1.98$ & $1 \cdot 13$ & $0 \cdot 75,1 \cdot 71$ & 1.49 & $0 \cdot 67,3 \cdot 31$ \\
\hline \multicolumn{7}{|l|}{ Mother's education } \\
\hline No education & 1.00 & Ref. & 1.00 & Ref. & 1.00 & Ref. \\
\hline Less than primary & $1 \cdot 21$ & $0.83,1 \cdot 75$ & $1 \cdot 32$ & $0.91,1.90$ & $1 \cdot 24$ & $0 \cdot 59,2 \cdot 60$ \\
\hline Primary & 0.99 & $0.68,1.44$ & $1 \cdot 04$ & $0.71,1 \cdot 51$ & 0.43 & $0 \cdot 15,1 \cdot 27$ \\
\hline Middle school & $1 \cdot 16$ & $0.89,1.53$ & $1 \cdot 34 t$ & $1 \cdot 03,1 \cdot 75$ & $1 \cdot 23$ & $0.72,2.09$ \\
\hline High school and above & $1 \cdot 25$ & $0.80,1.95$ & $1 \cdot 19$ & $0 \cdot 80,1 \cdot 78$ & 0.99 & $0.49,2 \cdot 02$ \\
\hline \multicolumn{7}{|l|}{ Mother's employment } \\
\hline \multicolumn{7}{|l|}{ Currently working } \\
\hline No & $1 \cdot 00$ & Ref. & $1 \cdot 00$ & Ref. & $1 \cdot 00$ & Ref. \\
\hline Yes & 0.93 & $0.69,1.26$ & 0.84 & $0 \cdot 63,1 \cdot 14$ & 0.77 & $0.42,1 \cdot 39$ \\
\hline \multicolumn{7}{|l|}{ Works away from home } \\
\hline No & 1.00 & Ref. & 1.00 & Ref. & 1.00 & Ref. \\
\hline Yes & $1 \cdot 33 \ddagger$ & $0.98,1 \cdot 81$ & $1 \cdot 24$ & $0.91,1.67$ & $1 \cdot 45$ & $0 \cdot 80,2 \cdot 64$ \\
\hline \multicolumn{7}{|l|}{ Other variables } \\
\hline \multicolumn{7}{|l|}{ Gender } \\
\hline Female & 1.00 & Ref. & $1 \cdot 00$ & Ref. & $1 \cdot 00$ & Ref. \\
\hline Male & 1.02 & $0.85,1.23$ & 0.96 & $0 \cdot 81,1 \cdot 15$ & $1 \cdot 14$ & $0.82,1.57$ \\
\hline \multicolumn{7}{|l|}{ Had diarrhoea in last week } \\
\hline No & 1.00 & Ref. & $1 \cdot 00$ & Ref. & $1 \cdot 00$ & Ref. \\
\hline Yes & 1.03 & $0 \cdot 81,1 \cdot 30$ & $0 \cdot 82 \ddagger$ & $0.65,1.03$ & $0.64 \ddagger$ & $0.40,1.01$ \\
\hline No. of siblings aged $<3$ years & $0 \cdot 79+$ & $0.64,0.98$ & $0.78 t$ & $0.63,0.97$ & $0.62 t$ & $0.40,0.95$ \\
\hline Child was first-born & & & & & & \\
\hline No & $1 \cdot 00$ & Ref. & $1 \cdot 00$ & Ref. & $1 \cdot 00$ & Ref. \\
\hline Yes & 1.09 & $0 \cdot 87,1 \cdot 36$ & 0.94 & $0 \cdot 76,1 \cdot 15$ & $0 \cdot 70 \ddagger$ & $0.48,1.01$ \\
\hline Low birth weight - subjective & & & & & & \\
\hline No & 1.00 & Ref. & 1.00 & Ref. & 1.00 & Ref. \\
\hline Yes & 0.93 & $0.64,1.35$ & $0 \cdot 82$ & $0.57,1 \cdot 17$ & $1 \cdot 18$ & $0.59,2 \cdot 36$ \\
\hline Rural area & & & & & & \\
\hline No & $1 \cdot 00$ & Ref. & $1 \cdot 00$ & Ref. & $1 \cdot 00$ & \\
\hline Yes & $0 \cdot 85$ & $0.67,1.07$ & $0 \cdot 83 \ddagger$ & $0.67,1.04$ & $1 \cdot 07$ & $0 \cdot 73,1 \cdot 57$ \\
\hline Observations & 2209 & & 2210 & & 2210 & \\
\hline
\end{tabular}

Ref., reference category.

Odds ratio with $95 \%$ confidence interval from the multivariate logistic regression models, stratified by WHO feeding guidelines, conditional on socio-economic variables.

All the covariates listed in the table were included for each model.

* Significant at $1 \%$ level.

+Significant at $5 \%$ level.

¥Significant at $10 \%$ level.

(including green leafy vegetables); eggs, meat, poultry, fish and shellfish (and organ meats)). Frequency of reading newspapers and magazines increased the likelihood that mothers gave complementary food and offered it at least twice daily and from at least three food groups $(\mathrm{OR}=1 \cdot 30$, $1 \cdot 21$ and $1 \cdot 18$, respectively). 
Table 4 Information and adequate feeding practices (9-18 months)

\begin{tabular}{|c|c|c|c|c|c|c|}
\hline & \multicolumn{2}{|c|}{ Complementary feeding (yes) } & \multicolumn{2}{|c|}{ Three or more times daily } & \multicolumn{2}{|c|}{ Four or more food groups } \\
\hline & \multicolumn{2}{|c|}{ Model 4} & \multicolumn{2}{|c|}{ Model 5} & \multicolumn{2}{|c|}{ Model 6} \\
\hline & OR & $95 \% \mathrm{Cl}$ & OR & $95 \% \mathrm{Cl}$ & OR & $95 \% \mathrm{Cl}$ \\
\hline \multicolumn{7}{|c|}{ Mother's exposure to health-care professionals } \\
\hline \multicolumn{7}{|c|}{ Had antenatal check up } \\
\hline No & $1 \cdot 00$ & Ref. & 1.00 & Ref. & $1 \cdot 00$ & Ref. \\
\hline Yes & $18 \cdot 82^{\star}$ & $12 \cdot 24,28 \cdot 95$ & $0.03^{*}$ & $0.01,0.07$ & $1 \cdot 89 \ddagger$ & $0.92,3.92$ \\
\hline \multicolumn{7}{|l|}{ Advised on nutrition (antenatal) } \\
\hline No & $1 \cdot 00$ & Ref. & $1 \cdot 00$ & Ref. & $1 \cdot 00$ & Ref. \\
\hline Yes & $1 \cdot 10$ & $0 \cdot 91,1 \cdot 33$ & $1 \cdot 03$ & $0 \cdot 92,1 \cdot 15$ & $1 \cdot 49^{*}$ & $1 \cdot 25,1 \cdot 78$ \\
\hline \multicolumn{7}{|c|}{ Met with health-care worker in last 3 months } \\
\hline No & $1 \cdot 00$ & Ref. & $1 \cdot 00$ & Ref. & $1 \cdot 00$ & Ref. \\
\hline Yes & $1 \cdot 20 \ddagger$ & $1 \cdot 00,1 \cdot 45$ & $1 \cdot 01$ & $0 \cdot 91,1 \cdot 12$ & $1 \cdot 13$ & $0 \cdot 97,1 \cdot 32$ \\
\hline \multicolumn{7}{|l|}{ Discussed nutrition and health } \\
\hline No & $1 \cdot 00$ & Ref. & $1 \cdot 00$ & Ref. & $1 \cdot 00$ & Ref. \\
\hline Yes & 0.91 & $0 \cdot 47,1 \cdot 78$ & $2 \cdot 34^{*}$ & $1 \cdot 58,3 \cdot 47$ & $1 \cdot 88^{*}$ & $1 \cdot 20,2 \cdot 94$ \\
\hline \multicolumn{7}{|l|}{ Exposure to media } \\
\hline Watched television & $1 \cdot 17^{\star}$ & $1 \cdot 07,1 \cdot 27$ & $1 \cdot 02$ & $0.98,1.08$ & $1 \cdot 01$ & $0.94,1.09$ \\
\hline Listened to radio & $1 \cdot 11+$ & $1 \cdot 02,1 \cdot 21$ & $1 \cdot 13^{*}$ & $1 \cdot 09,1 \cdot 19$ & $1 \cdot 12^{*}$ & $1 \cdot 05,1 \cdot 19$ \\
\hline Read newspaper or magazine & $1 \cdot 04$ & $0 \cdot 91,1 \cdot 20$ & $1 \cdot 07 \ddagger$ & $1 \cdot 00,1 \cdot 14$ & $1 \cdot 12^{*}$ & $1 \cdot 03,1 \cdot 21$ \\
\hline \multicolumn{7}{|l|}{ Wealth index } \\
\hline Poorest & $1 \cdot 00$ & Ref. & 1.00 & Ref. & $1 \cdot 00$ & Ref. \\
\hline Poorer & 0.97 & $0 \cdot 76,1 \cdot 24$ & 0.94 & $0 \cdot 80,1 \cdot 11$ & $1 \cdot 49 t$ & $1 \cdot 06,2 \cdot 07$ \\
\hline Middle & 1.01 & $0 \cdot 77,1 \cdot 32$ & $1 \cdot 00$ & $0 \cdot 85,1 \cdot 19$ & $2 \cdot 00^{\star}$ & $1 \cdot 45,2 \cdot 77$ \\
\hline Richer & $0 \cdot 86$ & $0 \cdot 63,1 \cdot 18$ & 0.93 & $0 \cdot 77,1 \cdot 12$ & $2 \cdot 49^{\star}$ & $1 \cdot 77,3 \cdot 49$ \\
\hline Richest & $1 \cdot 00$ & $0.66,1.52$ & $1 \cdot 08$ & $0 \cdot 86,1 \cdot 36$ & $2 \cdot 71^{\star}$ & $1 \cdot 86,3 \cdot 96$ \\
\hline \multicolumn{7}{|l|}{ Mother's education } \\
\hline No education & $1 \cdot 00$ & Ref. & $1 \cdot 00$ & Ref. & $1 \cdot 00$ & Ref. \\
\hline Less than primary & $1 \cdot 35^{\star}$ & $0 \cdot 98,1 \cdot 87$ & $1 \cdot 25 \ddagger$ & $1 \cdot 02,1 \cdot 52$ & $1 \cdot 50 \ddagger$ & $1 \cdot 08,2 \cdot 07$ \\
\hline Primary & $1 \cdot 08$ & $0 \cdot 78,1 \cdot 49$ & 0.90 & $0 \cdot 73,1 \cdot 11$ & $1 \cdot 63 t$ & $1 \cdot 19,2 \cdot 22$ \\
\hline Middle school & $1 \cdot 74 t$ & $1 \cdot 35,2 \cdot 25$ & $1 \cdot 35 t$ & $1 \cdot 17,1 \cdot 56$ & $1 \cdot 61+$ & $1 \cdot 28,2 \cdot 03$ \\
\hline High school and above & $2 \cdot 69 t$ & $1 \cdot 67,4 \cdot 34$ & $1 \cdot 44 t$ & $1 \cdot 16,1 \cdot 80$ & $2 \cdot 06 t$ & $1 \cdot 52,2 \cdot 79$ \\
\hline \multicolumn{7}{|l|}{ Mother's employment } \\
\hline \multicolumn{7}{|l|}{ Currently working } \\
\hline No & $1 \cdot 00$ & Ref. & $1 \cdot 00$ & Ref. & $1 \cdot 00$ & Ref. \\
\hline Yes & $1 \cdot 42^{*}$ & $0.98,2 \cdot 06$ & $1 \cdot 44 \dagger$ & $1 \cdot 19,1 \cdot 74$ & $1 \cdot 51+$ & $1 \cdot 17,1 \cdot 94$ \\
\hline \multicolumn{7}{|l|}{ Works away from home } \\
\hline No & $1 \cdot 00$ & Ref. & $1 \cdot 00$ & Ref. & $1 \cdot 00$ & Ref. \\
\hline Yes & $0 \cdot 84$ & $0 \cdot 57,1 \cdot 25$ & $0 \cdot 84$ & $0.68,1.04$ & $0.64 t$ & $0.48,0.85$ \\
\hline Other variables & & & & & & \\
\hline Gender & & & & & & \\
\hline Female & $1 \cdot 00$ & Ref. & $1 \cdot 00$ & Ref. & $1 \cdot 00$ & Ref. \\
\hline Male & 0.98 & $0 \cdot 83,1 \cdot 16$ & $1 \cdot 05$ & $0 \cdot 96,1 \cdot 16$ & 0.98 & $0 \cdot 85,1 \cdot 12$ \\
\hline Had diarrhoea in last week & & & & & & \\
\hline No & $1 \cdot 00$ & Ref. & $1 \cdot 00$ & Ref. & $1 \cdot 00$ & Ref. \\
\hline Yes & $0 \cdot 77 \ddagger$ & $0.62,0.95$ & 0.94 & $0 \cdot 83,1 \cdot 08$ & 0.92 & $0 \cdot 75,1 \cdot 12$ \\
\hline No. of siblings aged $<3$ years & $0 \cdot 48+$ & 11.92 & $0 \cdot 48 t$ & 11.92 & $0.66 t$ & $2 \cdot 80$ \\
\hline Child was first born & & & & & & \\
\hline No & $1 \cdot 00$ & Ref. & $1 \cdot 00$ & Ref. & $1 \cdot 00$ & Ref. \\
\hline Yes & $0 \cdot 89$ & $0 \cdot 73,1.09$ & 0.94 & $0 \cdot 84,1 \cdot 05$ & 0.90 & $0 \cdot 77,1 \cdot 05$ \\
\hline Low birth weight - subjective & & & & & & \\
\hline No & $1 \cdot 00$ & Ref. & $1 \cdot 00$ & Ref. & $1 \cdot 00$ & Ref. \\
\hline Yes & $1 \cdot 32$ & $0 \cdot 91,1 \cdot 89$ & 0.94 & $0 \cdot 77,1 \cdot 14$ & $1 \cdot 45 t$ & $1 \cdot 11,1 \cdot 91$ \\
\hline Rural area & & & & & & \\
\hline & $1 \cdot 00$ & Ref. & $1 \cdot 00$ & Ref. & $1 \cdot 00$ & Ref. \\
\hline Yes & $0 \cdot 69+$ & $0 \cdot 55,0 \cdot 86$ & 0.94 & $0.83,1.06$ & $1 \cdot 24 \ddagger$ & $1 \cdot 05,1 \cdot 47$ \\
\hline Observations & 7027 & & 7027 & & 7027 & \\
\hline
\end{tabular}

Ref., reference category.

Odds ratio with $95 \%$ confidence interval from the multivariate logistic regression models, stratified by WHO feeding guidelines, conditional on socio-economic variables.

All the covariates listed in the table were included for each model.

${ }^{*}$ Significant at $10 \%$ level.

tSignificant at $1 \%$ level.

¥Significant at $5 \%$ level.

Wealth and formal education were not found to be significant determinants of adequate feeding practices for infants in the age group 6-8 months (Table 3). Number of siblings younger than 3 years of age was found to be significant, and decreased the likelihood of timely introduction of complementary food, minimum meal 
frequency, as well as food diversity $(\mathrm{OR}=0 \cdot 79,0 \cdot 78$ and $0 \cdot 62$, respectively).

Even for children in the age group 9-18 months, nutritional information received from health professionals was a significant and important determinant of sound feeding practices. As shown in Table 4, mothers were more likely to offer food from four or more food groups if they had received nutritional advice during antenatal check-ups $(\mathrm{OR}=1.49)$ than if they had not. Mothers who discussed nutrition during their meetings with a health professional were more likely to offer food at least three times daily and from four or more food groups $(\mathrm{OR}=2 \cdot 34$ and 1.88 , respectively) than were mothers who did not discuss nutrition. As in the $6-8$ months age group, frequency of listening to the radio was the most important contact with a media source for obtaining information; it increased the likelihood of mothers offering complementary food, offering food at least three times daily and from four or more food groups (OR $=$ $1 \cdot 11,1 \cdot 13$ and $1 \cdot 12$, respectively).

For children in the age group 9-18 months, unlike for the younger infants, mothers' formal education and wealth index were positively associated with sound feeding practices, and the magnitude of the effect increased with an increase in education level and wealth index (Table 4). Compared with mothers from the poorest households, mothers from poorer, middle, richer and richest households were more likely to offer their children food from four or more food groups (OR $=1 \cdot 49,2 \cdot 00,2 \cdot 49$ and 2.71, respectively). Compared with mothers with no education, mothers with less than primary, primary, middle school and high school education were more likely to offer food from four or more food groups (OR $=1.50,1.63,1.61$ and $2 \cdot 06$, respectively). Compared with mothers with no education, mothers with less than primary, middle school and high school education were also more likely to offer food three or more times daily $(\mathrm{OR}=1 \cdot 25,1.35$ and $1 \cdot 44$, respectively).

The other important factors of sound feeding practices were the mother's work status, the number of siblings and if the child was the first-born (Table 4). Working mothers were more likely to follow good feeding practices compared with mothers without formal employment. However, mothers who worked outside the home were less likely to offer their child food from four or more food groups $(\mathrm{OR}=0.64)$ compared with mothers who worked from home. As expected, more siblings less than 3 years of age lowered the likelihood that a child would be offered complementary food, be fed three times or more daily and from four or more food groups ( $\mathrm{OR}=0 \cdot 48,0 \cdot 48$ and $0 \cdot 66$, respectively). Recent diarrhoeal episode reduced the likelihood of mothers offering complementary food in this age group $(\mathrm{OR}=0 \cdot 77)$. Mothers in rural areas were less likely to introduce complementary food on time $(\mathrm{OR}=0 \cdot 69)$, but more likely to offer food from four or more food groups $(\mathrm{OR}=1 \cdot 24)$.

\section{Discussion and conclusion}

The current work demonstrates that a lack of nutritional information is a major barrier to the following of sound nutritional practices in India. Across all wealth classes, mothers who had discussed nutrition with their health workers were more likely to follow IYCF guidelines while feeding their child. Nutritional information and advice offered by anganwadi workers and other health professionals significantly improved the feeding practices.

These results also highlight the success of anganwadi centres (set up by the Indian Government under the Integrated Child Development Services) in achieving one of their major goals: nutritional and health education for women in the age group of 15-49 years. Anganwadis are the primary instrument against child malnutrition, with the main aim of educating mothers and caregivers in following appropriate feeding practices. They give nutritional advice on appropriate and adequate complementary feeding practices like introducing complementary food at the age of 6 months, and also give guidance on appropriate preparation of local foods for children ${ }^{(20)}$. In the current study, nutritional advice from anganwadi workers was shown to significantly change the behaviour of mothers in following the IYCF guidelines.

Media, especially frequency of listening to the radio, was positively related to sound feeding practices; this is not surprising as mass media is an important channel for the Ministry of Women and Child Development (Government of India) to dispense nutrition information. Major steps were taken in 2006 with the launch of advertising campaigns on nutritional issues: a thirtyepisode sponsored radio programme broadcast in twenty regional languages and wall calendars on nutrition were widely distributed all across the country ${ }^{(33)}$.

It is not wealth that seems to determine whether a child in the age group 6-8 months is offered complementary solid or semi-solid food, but information on nutrition that plays a significant role; the same holds true for whether a child in the age group 6-8 months is offered food at least two times daily and from three or more food groups. This emphasizes the need to counsel and educate mothers about children's nutritional needs and appropriate feeding practices as per the Indian National Guidelines on IYCF. Wealth was found to be significantly important in determining how many times the child is fed in a day and whether the child is offered food from four or more food groups for children in the age group 9-18 months; however, even after controlling for wealth and formal education, nutritional information was found to be a significant determinant also for this age group.

In a developing country like India, the mother plays the central role in childrearing. Thus, the mother's employment was found to be a significant determinant. A working mother was more likely to follow good feeding practices than a mother who does not have formal 
employment. Since the mother's education is already controlled for, the result suggests that a working mother might be more aware because of her social interactions or might be more proactive in obtaining information. However, if a mother had to leave home to work outside, the child was less likely to be offered appropriate food compared with when a mother worked from home; this might be the direct result of having less time to feed the child and might also reflect the inadequacy of the people substituting for the mother in childrearing while she is at work ${ }^{(34)}$.

There is empirical evidence of the impact of health information in other health contexts related to hygiene, oral rehydration therapy and other spheres of health care on the health status of people ${ }^{(35-38)}$. The present study shows that the same is true of nutritional information in Indian child feeding practices. Among mothers who met with health professionals, those who received nutritional advice were more likely to follow better IYCF practices when compared with those who met them but did not receive advice. Some earlier studies have failed to show a significant improvement in IYCF practices following nutritional education due to a small sample size, the content of the education or the design of the intervention $^{(39-41)}$. Other nutritional intervention studies that coupled nutritional education with provision of complementary food, fortification of complementary food or food supplements have found nutritional education to be effective in improving feeding practices ${ }^{(41,42)}$.

In India, child malnutrition is widespread and affects up to 75 million children under the age of 3 years. The present work demonstrates that poverty is an implied contributor, but it is not the sole reason for this situation. Even among the wealthiest category (top quintile) of households in the survey, only $40 \%$ of children received adequate food as per the WHO guidelines. If information about childhood feeding practices is a key obstacle to improved nutrition, then the prognosis is hopeful; it is easier to resolve the problem of scarce information than to resolve the problem of acute poverty. Ultimately, extensive intervention is needed to educate families about proper infant feeding practices. Traditional and cultural beliefs related to early childhood feeding are also a strong barrier to eradicating malnutrition $^{(40,43,44)}$ that needs to be addressed at the local level. Advice and counselling by health workers should not only educate mothers about their child's nutritional needs, but also address the restricted traditional beliefs that might be acting as a barrier to sound feeding practices. This should be done at multiple levels, and nutrition education should also be made an integral part of primary and secondary education.

Since nutritional advice during antenatal check-ups had the biggest impact on feeding practices, early childhood nutrition advice should be made available at every public health facility, not only for mothers and pregnant women, but also for women of childbearing age as well as other caregivers.

\section{Acknowledgements}

Sources of funding: This research received no specific grant from any funding agency in the public, commercial or not-for-profit sectors. Conflicts of interest: There are no conflicts of interest. Acknowledgements: The author would like to thank Associate Professor Milind Kandlikar and Assistant Professor Judy A. McLean for helpful suggestions in writing the manuscript.

\section{References}

1. Subramanyam MA, Kawachi I, Berkman LF et al. (2010) Socioeconomic inequalities in childhood undernutrition in India: analyzing trends between 1992 and 2005. PLOS ONE 5, e11392.

2. Pelletier DL (1994) The relationship between child anthropometry and mortality in developing countries: implications for policy, programs and future research. J Nutr 124, 10 Suppl., 2047S-2081S.

3. Martorell R, Ramakrishnan U, Schroeder DG et al. (1996) Reproductive performance and nutrition during childhood. Nutr Rev 54, 4 Pt 2, S15-S21.

4. Li H, Stein AD, Barnhart HX et al. (2003) Associations between prenatal and postnatal growth and adult body size and composition. Am J Clin Nutr 77, 1498-1505.

5. Osmond C, Barker DJ, Winter PD et al. (1993) Early growth and death from cardiovascular disease in women. BMJ 307, 1519-1524.

6. Eriksson JG, Forsen T, Tuomilehto J et al. (2001) Early growth and coronary heart disease in later life: longitudinal study. BMJ 322, 949-953.

7. Haas JD, Martinez EJ, Murdoch E et al. (1995) Nutritional supplementation during the preschool years influences body size and composition of Guatemalan adolescents. J Nutr 125, 4 Suppl., 1078S-1089S.

8. Horta BL, Gigante DP, Osmond C et al. (2009) Intergenerational effect of weight gain in childhood on offspring birthweight. Int J Epidemiol 38, 724-732.

9. International Institute for Population Sciences \& Macro International (2007) National Family Health Survey (NFHS-3), 2005-06, India: Key Findings. http://www.measuredhs.com/ pubs/pdf/SR128/SR128.pdf (accessed February 2011).

10. Martorell R, Rivera J, Kaplowitz H et al. (1992) Long-term consequences of growth retardation during early childhood. In Human Growth: Basic and Clinical Aspects, pp. 143-149 [M Hernandez and J Argente, editors]. Amsterdam: Elsevier Science.

11. Brown KH (1991) The importance of dietary quality versus quantity for weanlings in less developed countries: a framework for discussion. Food Nutr Bull 13, 86-94.

12. World Health Organization, UNICEF \& Partners (2010) Indicators for Assessing Infant and Young Child Feeding Practices, Part 3: Country Profiles. Geneva: WHO.

13. Saha KK, Frongillo EA, Alam DS et al. (2008) Appropriate infant feeding practices result in better growth of infants and young children in rural Bangladesh. Am J Clin Nutr 87, 1852-1859.

14. Dewey KG, Finley DA \& Lonnerdal B (1984) Breast milk volume and composition during late lactation (7-20 months). J Pediatr Gastroenterol Nutr 3, 713-720.

15. Laskey MA, Prentice A, Shaw J et al. (1990) Breast-milk calcium concentrations during prolonged lactation in British and rural Gambian mothers. Acta Paediatr 79, 507-512.

16. Brown KH, Dewey KG \& Allen LH (1998) Complementary Feeding of Young Children in Developing Countries: A Review of Current Scientific Knowledge. New York: UNICEF. 
17. Arimond M \& Ruel MT (2004) Dietary diversity is associated with child nutritional status: evidence from 11 demographic and health surveys. J Nutr 134, 2579-2585.

18. Sawadogo PS, Martin-Prevel Y, Savy M et al. (2006) An infant and child feeding index is associated with the nutritional status of 6- to 23-month-old children in rural Burkina Faso. J Nutr 136, 656-663.

19. World Health Organization, UNICEF \& Partners (2010) Indicators for Assessing Infant and Young Child Feeding Practices, Part 2: Measurement. Geneva: WHO.

20. Ministry of Women and Child Development (2006) National Guidelines on Infant and Young Child Feeding. New Delhi: Food and Nutrition Board, Government of India.

21. India Ministry of Health and Family Welfare (2003) Integrated Management of Neonatal and Childhood Illness. Training Module of Health Workers, 74-5. New Delhi: Ministry of Health and Family Welfare, Government of India.

22. Dewey KG (2001) The challenges of promoting optimal infant growth. $J$ Nutr 131, 1879-1880.

23. Lahariya C \& Khandekar J (2007) How the findings of national family health survey-3 can act as a trigger for improving the status of anemic mothers and undernourished children in India: a review. Indian J Med Sci 61, 535-544.

24. Caulfield LE, Huffman SL \& Piwoz EG (1999) Interventions to improve intake of complementary foods by infants 6 to 12 months of age in developing countries: impact on growth and on the prevalence of malnutrition and potential contribution to child survival. Food Nutr Bull 20, 183-200.

25. Svedberg P (2000) Poverty and Undernutrition: Theory, Measurement, and Policy. Oxford: Oxford University Press.

26. Pryer JA, Rogers S \& Rahman A (2004) The epidemiology of good nutritional status among children from a population with a high prevalence of malnutrition. Public Health Nutr 7, 311-317.

27. Fotso JC \& Kuate-Defo B (2006) Household and community socioeconomic influences on early childhood malnutrition in Africa. J Biosoc Sci 38, 289-313.

28. Subramanyam MA, Kawachi I, Berkman LF et al. (2010) Socioeconomic inequalities in childhood undernutrition in India: analyzing trends between 1992 and 2005. PLOS ONE 5, e11392.

29. Webb AL, Sellen DW, Ramakrishnan U et al. (2009) Maternal years of schooling but not academic skills is independently associated with infant-feeding practices in a cohort of rural Guatemalan women. J Hum Lact 25, 297-306.

30. Liaqat P, Rizvi MA, Qayyum A et al. (2007) Association between complementary feeding practice and mother's education status in Islamabad. J Hum Nutr Diet 20, 340-344.

31. Saha KK, Frongillo EA, Alam DS et al. (2008) Household food security is associated with infant feeding practices in rural Bangladesh. J Nutr 138, 1383-1390.
32. International Institute for Population Sciences \& ORC Macro (2007) National Family Health Survey (NFHS-3), 2005-06: India, Volume I. Mumbai: IIPS.

33. Ministry of Women and Child Development, Government of India (2006) Highlights of Nutrition Initiatives of FNB and MWCD during the last two years in the context of achieving Food and Nutrition Security in the country. http://motherchildnutrition.org/india/pdf/mon-highlights-ofnutrition-initiatives.pdf (accessed November 2011).

34. Abbi R, Christian P, Gujral S et al. (1991) The impact of maternal work on the nutrition and health status of children. Food Nutr Bull 13, 20-24.

35. Jalan J \& Somanthan E (2004) The importance of being informed: experimental evidence on demand for environmental quality. J Dev Econ 87, 14-28.

36. Santosham M, Chandran A, Fitzwater S et al. (2010) Progress and barriers for the control of diarrhoeal disease. Lancet 376, 63-67.

37. Siwach M (2009) Impact of health education programme on the knowledge and practices of school children regarding personal hygiene in rural Panipat. Int $J E d u c$ Sci 1, 115-118.

38. Sherwani J, Ali N, Mirza S et al. (2007) HealthLine: speechbased access to health information by low-literate users. In Proceedings of the 2nd IEEE/ACM International Conference on Information and Communications Technologies and Development (ICTD2007), Bangalore, India, 15-16 December 2007, pp. 131-139.

39. Bhandari N, Bahl R, Nayyar B et al. (2001) Food supplementation with encouragement to feed it to infants from 4 to 12 months of age has a small impact on weight gain. J Nutr 131, 1946-1951.

40. Palwala M, Sharma S, Udipi SA et al. (2009) Nutritional quality of diets fed to young children in urban slums can be improved by intensive nutrition education. Food Nutr Bull 30, 317-326.

41. Dewey KG \& Adu-Afarwuah S (2008) Systematic review of the efficacy and effectiveness of complementary feeding interventions in developing countries. Matern Child Nutr 4, Suppl. 1, 24-85.

42. Aamer I, Yakoob MY \& Bhutta ZA (2011) Impact of maternal education about complementary feeding and provision of complementary foods on child growth in developing countries. BMC Public Health 11, Suppl. 3, S25.

43. Bhandari NR \& Patel GP (1973) Dietary and feeding habits of infants in various socio-economic groups. Indian Pediatr 10, 233.

44. Guldan GS, Fan HC, Ma X et al. (2000) Culturally appropriate nutrition education improves infant feeding and growth in rural Sichuan. China J Nutr 130, 1204-1211. 